\title{
SOME DEGENERATIONS OF A COMPACT RIEMANN SURFACE OF GENUS 4
}

\author{
BY AARON LEBOWITZ
}

Communicated by F. W. Gehring, September 30, $1974^{1}$

ABSTRACT. A number of degenerations of a compact hyperelliptic Riemann surface of genus 4 are studied, using theta function techniques.

1. Let $(S, \Gamma, \Delta)$ be a hyperelliptic Riemann surface of genus 4 with a canonical homology basis. Then $S$ has a representation as a two-sheeted cover of the sphere with ten branch points. We can arrange for the surface to have branch points over $0,1, \infty, 1 / \lambda_{1}, 1 / \lambda_{2}, \cdots, 1 / \lambda_{7}$, where its real branch points other than 0 and 1 (if any) are all greater than 1 and in ascending order (see [2]). We obtain a concrete representation of $(S, \Gamma, \Delta)$ which we henceforth assume is that illustrated in Figure 1.

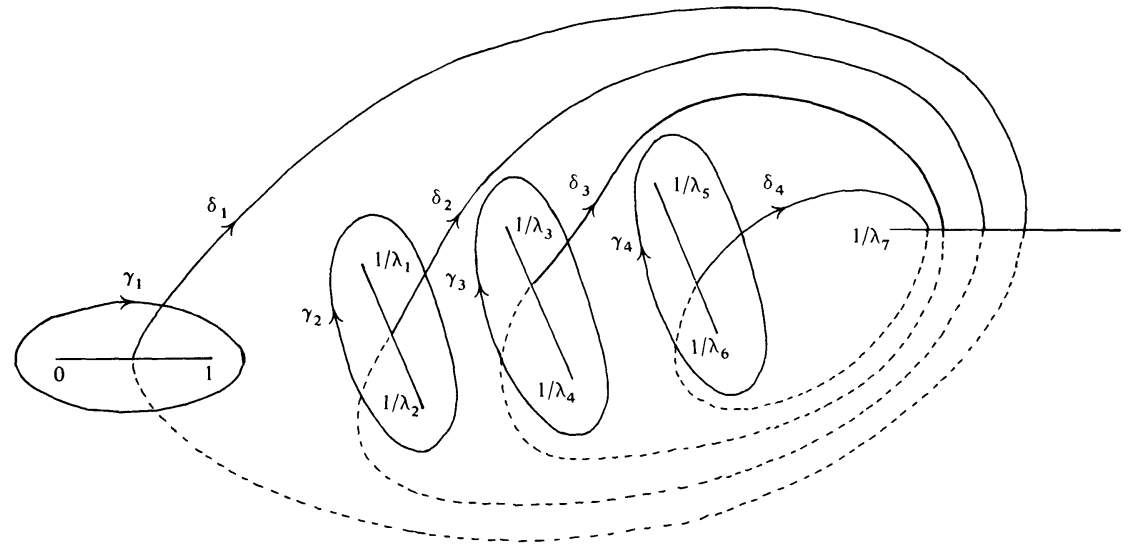

FIGURE 1

For all introductory material we refer the reader to [1], [2] and [3]. There are, for $(S, \Gamma, \Delta)$, precisely ten even theta functions which vanish at the origin (see [1]). We will insist in our degeneration process that these even theta functions remain zero at 0 , thus insuring that the surface remains hyperelliptic.

AMS (MOS) subject classifications (1970). Primary 14H15, 30A46, 32G 15.

1 Originally received July 23, 1974. 
THEOREM 1. Let $(S, \Gamma, \Delta)$ be as described in Figure 1. Then the branch points $1 / \lambda_{i}, i=1,2, \cdots, 7$, are computed as the following quotients of products of Riemann theta constants, where we suppress the dependency of the constants on the matrix $\pi$ :

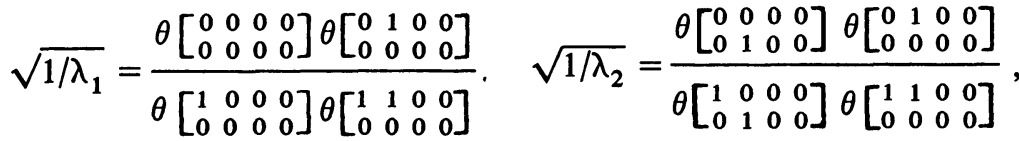

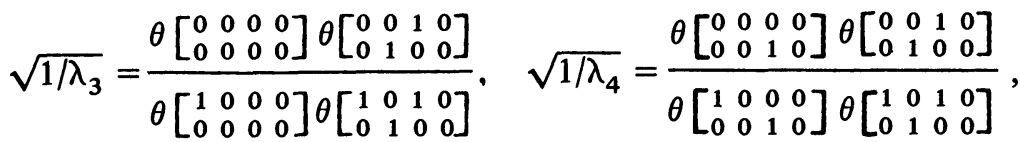

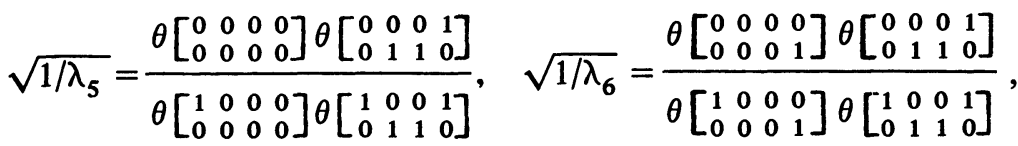

$$
\begin{aligned}
& \sqrt{1 / \lambda_{7}}=\frac{\theta\left[\begin{array}{llll}
0 & 0 & 0 & 0 \\
0 & 0 & 0 & 0
\end{array}\right] \theta\left[\begin{array}{llll}
0 & 0 & 0 & 0 \\
0 & 1 & 1 & 1
\end{array}\right]}{\theta\left[\begin{array}{llll}
1 & 0 & 0 & 0 \\
0 & 0 & 0 & 0
\end{array}\right] \theta\left[\begin{array}{llll}
1 & 0 & 0 & 0 \\
0 & 1 & 1 & 1
\end{array}\right]}
\end{aligned}
$$

Proof. See [2] for the proof of a similar theorem.

2. Let $\left(\pi_{i j}\right)$ be a period matrix in $\S_{4}$, which is, therefore, not equivalent to a diagonal matrix. For each degeneration we shall assume that we vary (if necessary) a sufficient number of the periods so that the surface remains hyperelliptic, while certain sets of the periods are allowed to tend to zero. We also assume that the limiting blocks of periods which remain are not equivalent to diagonal matrices and belong to hyperelliptic Riemann surfaces and that during the degeneration we are at no time at a matrix which is equivalent to a diagonal one.

We describe by means of a definition a number of degenerations of $(S, \Gamma, \Delta)$ with period matrix $\left(\pi_{i j}\right)$ where the appropriate degenerating periods are assumed sufficiently near zero and the seven moveable branch points are all far from the interval $[0,1]$.

Definition 1. (a) Let $\pi_{i j}, i=1,2, j=3,4$, tend to zero. We obtain in the limit two blocks,

$$
\left(\begin{array}{ll}
\pi_{11} & \pi_{12} \\
\pi_{12} & \pi_{22}
\end{array}\right) \text { and }\left(\begin{array}{ll}
\pi_{33} & \pi_{34} \\
\pi_{34} & \pi_{44}
\end{array}\right)
$$

We associate with these matrices, surfaces $S_{1}$ and $S_{2}$, each of genus 2 (see [2]).

(b) Let $\pi_{1 j}, j=2,3,4$ and $\pi_{2 k}, k=3,4$, tend to zero. We obtain 
three blocks, $\left(\pi_{11}\right),\left(\pi_{22}\right)$ and

$$
\left(\begin{array}{ll}
\pi_{33} & \pi_{34} \\
\pi_{34} & \pi_{44}
\end{array}\right)
$$

to which we associate surfaces $S_{3}, S_{4}$ of genus 1 and $S_{2}$ of genus 2 .

(c) Let $\pi_{i j}, i \neq j$, tend to zero. In the limit we obtain four blocks, $\left(\pi_{i i}\right)$, $i=1,2,3,4$. To each we associate a surface of genus 1 , say $S_{3}, S_{4}, S_{5}$, and $S_{6}$.

(d) Let $\pi_{4 j}, j=1,2,3$, tend to zero. We obtain two blocks $\left(\pi_{44}\right)$ and

$$
\left(\begin{array}{lll}
\pi_{11} & \pi_{12} & \pi_{13} \\
\pi_{12} & \pi_{22} & \pi_{23} \\
\pi_{13} & \pi_{23} & \pi_{33}
\end{array}\right) .
$$

We associate $S_{6}$ with $\left(\pi_{44}\right)$ and $S_{7}$ of genus 3 with the $3 \times 3$ block.

Henceforth, in referring to $S_{1}$, etc., the words "a suitable two-sheeted representation of" will be understood but omitted.

We have the following theorems, whose parts correspond to the various parts of Definition 1.

THEOREM 2. Let $(S, \Gamma, \Delta)$ be as in Figure 1. In each part of this theorem the limits obtained are the appropriate nonnormalized branch points of the appropriate surface $S_{1}$, etc.

(a) Let the period matrix for $S$ degenerate as in Definition 1(a). Then the branch points $1 / \lambda_{i}, i=1,2, \cdots, 7$, degenerate as follows:

$$
\begin{aligned}
& 1 / \lambda_{1} \rightarrow \frac{\theta^{2}\left[\begin{array}{ll}
0 & 0 \\
0 & 0
\end{array}\right] \theta^{2}\left[\begin{array}{ll}
0 & 1 \\
0 & 0
\end{array}\right]}{\theta^{2}\left[\begin{array}{ll}
1 & 0 \\
0 & 0
\end{array}\right] \theta^{2}\left[\begin{array}{ll}
1 & 1 \\
0 & 0
\end{array}\right]}, \quad 1 / \lambda_{2} \rightarrow \frac{\theta^{2}\left[\begin{array}{ll}
0 & 1 \\
0 & 0
\end{array}\right] \theta^{2}\left[\begin{array}{ll}
0 & 0 \\
0 & 1
\end{array}\right]}{\theta^{2}\left[\begin{array}{ll}
1 & 1 \\
0 & 0
\end{array}\right]^{2}\left[\begin{array}{ll}
1 & 0 \\
0 & 1
\end{array}\right]}, \\
& 1 / \lambda_{i} \rightarrow \frac{\theta^{2}\left[\begin{array}{ll}
0 & 0 \\
0 & 0
\end{array}\right] \theta^{2}\left[\begin{array}{ll}
0 & 0 \\
0 & 1
\end{array}\right]}{\theta^{2}\left[\begin{array}{ll}
1 & 0 \\
0 & 0
\end{array}\right] \theta^{2}\left[\begin{array}{ll}
1 & 0 \\
0 & 1
\end{array}\right]}, \quad i=3, \cdots, 7,
\end{aligned}
$$

where the period matrix for all the theta constants is

$$
\left(\begin{array}{ll}
\pi_{11} & \pi_{12} \\
\pi_{12} & \pi_{22}
\end{array}\right)
$$

(b) Let the period matrix degenerate as in Definition 1(b). Then the seven branch points coalesce as follows: 


$$
1 / \lambda_{i} \rightarrow \frac{\theta^{4}\left[\begin{array}{l}
0 \\
0
\end{array}\right]\left(0, \pi_{11}\right)}{\theta^{4}\left[\begin{array}{l}
1 \\
0
\end{array}\right]\left(0, \pi_{11}\right)}, \quad i=1, \cdots, 7 .
$$

(c) Same as (b), replace Definition 1(b) by Definition 1(c).

(d) Degenerate as in Definition 1(d). Then

$$
\begin{aligned}
1 / \lambda_{1} \rightarrow \frac{\theta^{2}\left[\begin{array}{lll}
0 & 0 & 0 \\
0 & 0 & 0
\end{array}\right] \theta^{2}\left[\begin{array}{lll}
0 & 1 & 0 \\
0 & 0 & 0
\end{array}\right]}{\theta^{2}\left[\begin{array}{lll}
1 & 0 & 0 \\
0 & 0 & 0
\end{array}\right] \theta^{2}\left[\begin{array}{lll}
1 & 1 & 0 \\
0 & 0 & 0
\end{array}\right]}, & 1 / \lambda_{2} \rightarrow \frac{\theta^{2}\left[\begin{array}{lll}
0 & 0 & 0 \\
0 & 1 & 0
\end{array}\right] \theta^{2}\left[\begin{array}{lll}
0 & 1 & 0 \\
0 & 0 & 0
\end{array}\right]}{\theta^{2}\left[\begin{array}{lll}
1 & 0 & 0 \\
0 & 1 & 0
\end{array}\right] \theta^{2}\left[\begin{array}{lll}
1 & 1 & 0 \\
0 & 0 & 0
\end{array}\right]}, \\
1 / \lambda_{3} \rightarrow \frac{\theta^{2}\left[\begin{array}{lll}
0 & 0 & 0 \\
0 & 0 & 0
\end{array}\right] \theta^{2}\left[\begin{array}{lll}
0 & 0 & 1 \\
0 & 1 & 0
\end{array}\right]}{\theta^{2}\left[\begin{array}{lll}
1 & 0 & 0 \\
0 & 0 & 0
\end{array}\right] \theta^{2}\left[\begin{array}{lll}
1 & 0 & 1 \\
0 & 1 & 0
\end{array}\right]}, & 1 / \lambda_{4} \rightarrow \frac{\theta^{2}\left[\begin{array}{lll}
0 & 0 \\
0 & 0 & 1
\end{array}\right] \theta^{2}\left[\begin{array}{lll}
0 & 1 & 1 \\
0 & 1 & 0
\end{array}\right]}{\theta^{2}\left[\begin{array}{lll}
1 & 0 & 0 \\
0 & 0 & 1
\end{array}\right] \theta^{2}\left[\begin{array}{lll}
1 & 0 & 1 \\
0 & 1 & 0
\end{array}\right]}, \\
1 / \lambda_{i} \rightarrow \frac{\theta^{2}\left[\begin{array}{lll}
0 & 0 & 0 \\
0 & 0 & 0
\end{array}\right] \theta^{2}\left[\begin{array}{lll}
0 & 0 & 0 \\
0 & 1 & 1
\end{array}\right]}{\theta^{2}\left[\begin{array}{lll}
1 & 0 & 0 \\
0 & 0 & 0
\end{array}\right] \theta^{2}\left[\begin{array}{lll}
1 & 0 & 0 \\
0 & 1 & 1
\end{array}\right]}, & i=5,6,7 .
\end{aligned}
$$

PROOF. The result is clear from the observation that the theta functions split when the period matrix splits into blocks along the major diagonal. For part (d), we note that the condition for hyperellipticity is that the one even theta with characteristic $\left[\begin{array}{lll}1 & 1 & 1 \\ 1 & 0 & 1\end{array}\right]$ be zero at the origin, which is indeed the case.

THEOREM 3. For each case below there exist transformations $z^{\prime}=a(\pi) z$ $+b(\pi), \pi=\left(\pi_{i j}\right)$ such that the transforms of the branch points have the indicated behavior in the limit as $\left(\pi_{i j}\right)$ splits according to Definition 1.

(a) The branch points $1 / \lambda_{i}^{\prime}, i=3, \cdots, 7$, tend to the finite branch points on $S_{2}$, while $0^{\prime}, 1^{\prime}, 1 / \lambda_{1}^{\prime}, 1 / \lambda_{2}^{\prime}$ all tend to $\infty$.

(b) $1 / \lambda_{1}^{\prime}=0,1 / \lambda_{2}^{\prime}=1$, and $1 / \lambda_{i}^{\prime}, i=3, \cdots, 7$, tend to the fourth branch point on $S_{4}$, while $0^{\prime}, 1^{\prime}$ tend to $\infty$.

(c) $1 / \lambda_{3}^{\prime}=0,1 / \lambda_{4}^{\prime}=1$, and $1 / \lambda_{i}^{\prime}, i=5,6,7$, tend to the fourth branch point on $S_{5}$, while $0^{\prime}, 1^{\prime}, 1 / \lambda_{1}^{\prime}, 1 / \lambda_{2}^{\prime}$ all tend to $\infty$. $1 / \lambda_{5}^{\prime}=0,1 / \lambda_{6}^{\prime}=1$ and $1 / \lambda_{7}^{\prime}$ tends to the fourth branch point on $S_{6}$, while $0^{\prime}, 1^{\prime}, 1 / \lambda_{i}^{\prime}, i=1, \cdots, 4$, all tend to $\infty$.

(d) See the last sentence of part (c).

\section{REFERENCES}

1. H. M. Farkas, Period relations for hyperelliptic Riemann surfaces, Israel J. Math. 10 (1971), 289-301. 
2. A. Lebowitz, Degeneration of a compact Riemann surface of genus 2 , Israel J. Math. 12 (1972), 223-236.

3. H. E. Rauch and H. M. Farkas, Theta constants of two kinds on a compact Riemann surface of genus 2, J. Analyse Math. 23 (1970), 381-407. MR 43 \#503.

DEPARTMENT OF MATHEMATICS, HERBERT LEHMAN COLLEGE (CUNY), BRONX, NEW YORK 10468

DEPARTMENT OF MATHEMATICS, BEN GURION UNIVERSITY OF THE NEGEV, BEERSHEBA, ISRAEL 